\title{
A MULTI-SENSOR PARAMETRIC IDENTIFICATION PROCEDURE IN THE FREQUENCY DOMAIN FOR THE REAL-TIME SURVEILLANCE OF FLUTTER
}

\author{
Pierre Vacher ${ }^{*, 1}$ Alain Bucharles ${ }^{*, 1}$ \\ * ONERA, Flight Dynamics and Control System \\ Department, 2 av. Edouard Belin, 31055 Toulouse cedex, \\ France-Email : Pierre.Vacher@onera.fr, \\ Alain.Bucharles@cert.fr - Tel : +33 5.62.25.27.63 , \\ +33 5.62.25.27.64 - Fax: +33 5.62.25.25.64.
}

\begin{abstract}
:
Flutter flight tests is a crucial and feared phase of the flight test program of a new aircraft. The specific operational context of flutter surveillance implies the development of automatic and reliable tools operating in real-time. At ONERA, we recently developed a toolbox dedicated to data processing for flutter tests. It was used for the latest Airbus aircraft from the A340-600 up to the A380.

In this article, we present the main procedure of the toolbox : the identification routine together with the graphical interfaces that were designed to help the operator to get a rapid knowledge of the identification results.
\end{abstract}

Keywords: Identification, Algorithms, Polynomials, Transfer functions, Frequency domain

\section{INTRODUCTION}

Because of their size, their architecture and their light structure, the most recent aircraft of the Airbus company exhibit a high structural flexibility. This tendency is also accompanied by a much greater density of the aeroelastic modes, especially for four-engine configurations. The modal analysis therefore becomes increasingly complex because the proximity of the modes and their greater number.

Up until the launch of the Airbus A340-600, the flutter surveillance was based on the tools that were developed at ONERA in 1987 for the tests of the Airbus A320. These tools relied on a monotransfer modal analysis, i.e. performed independently on each measurement. The modal synthe-

\footnotetext{
1 We would like to thank Anne Pin-Belloc, Cécile Daudet, Jean Roubertier, Frédéric Dessillons at Airbus for their constructive cooperation during the MEFAS project.
}

sis, i.e. the pairing of the modes identified for each sensor was carried out by the human operator. He was later helped by an expert system.

As the Airbus A340-600 was expected to present a much higher modal density, these tools were judged unsuitable for this aircraft. A research program named MEFAS (Méthodes et Exploitation des essais de Flottement de l'Avion Souple) was then launched as a cooperation between Airbus and ONERA in the years 1999- 2001. These studies resulted in the development of a set of new tools dedicated to flutter testing. They were implemented in the telemetry center of Airbus and used for the flight tests of several Airbus aircraft : A340-600 (April 2001), A318 (January 2002), A340-500 (February 2002), A380 (April 2005). This article is devoted to the presentation of the main tool of MEFAS : the modal identification. 
Historically at Airbus, the flutter tests consisted in exciting the aircraft structure with sine-sweep excitations. The transfers associated with the measurements were computed using a classical method due to P. Welch (Welch, 1967). But the economical urge for shorter flight tests leads more and more to the use of pulse excitations. The test duration is then reduced from 2 minutes to about 10 seconds. In this case, the signal length is too short to perform a spectrum estimation.

Therefore, the new identification procedure had to be able to process as input data both estimated transfers and raw frequential measurements. As it is designed to operate in real-time, it must also be entirely automatic and rapid. Finally, to meet the requirements about the greater modal density, we opted for a multi-sensor approach in order to get the maximum advantage of the modal information in the measurements.

The frequency domain is particularly appropriate to the test conditions since we can focus on the sole frequency band of interest. The amount of data to be processed is also greatly reduced resulting in improved computation times. Finally the people at Airbus were familiar with a frequency approach and several tools were already available.

The orientation towards a parametric approach was decided by a preliminary analysis of the available identification techniques. Subspace approaches were discarded based on several considerations: their lack of maturity, the generation of numerous spurious modes, few methods available in the frequency domain, the difficulty to automatize them. As shown in the article, a parametric approach proves quite adequate to the specifications of flutter surveillance. It also constitutes a graceful extension of the procedure in operation until then at Airbus.

This paper is organized as followed. The first section is devoted to the presentation of the new identification algorithm. In the second section, we discuss how the parametrisation of the model can be optimized for the best numerical performance. In the third section, we describe the tools developed to monitor the identification process on two aspects : the determination of the order of the identified model, the analysis of its characteristics.

\section{THE IDENTIFICATION PROCEDURE}

\subsection{Description of the problem}

The goal of identification is to find the best fit between, on the one hand, a parametric model and, on the other hand, either estimated transfers or frequential measurements. As we opted for an approach in the frequency domain, we will only consider, in this article, the data at specific frequencies of interest $f$ within a frequency set denoted $\mathscr{F}$.

Since the aircraft is excited by a unique signal, it can be modeled by a single input system. We adopted the following parametrisation for the system transfer function:

$$
\mathrm{H}(s, \theta)=\frac{\mathrm{N}(s, \theta)}{\mathrm{d}(s, \theta)}
$$

where

- the denominator $\mathrm{d}(s, \theta)$ is a polynomial of degree $n_{d}$

- the numerator $\mathrm{N}(s, \theta)$ is a polynomial vector of size $n_{y} \times 1$ and of degree $n_{d}$

- $s$ is the Laplace transform variable

- $\theta$ is the vector of dimension $\left(n_{y}+1\right)\left(n_{d}+1\right)$ which gathers the coefficients of the numerator and denominator. It can be subdivided into:

$$
\theta=\left(\begin{array}{c}
\theta_{1} \\
\vdots \\
\theta_{n_{y}} \\
\theta_{\mathrm{d}}
\end{array}\right)
$$

where $\theta_{l}$ are the coefficients of the $l$ th row in the numerator and $\theta_{\mathrm{d}}$ those of the denominator.

At this stage, we suppose the system order $n_{d}$ fixed. The determination of $n_{d}$ will be addressed later in section 4.1. In order to define a nondegenerated problem, the degree zero coefficient of the denominator is set to the value 1 . In the rest of the article, we only consider the values of $\mathrm{H}(s, \theta)$ for the frequencies $f$ in the set $\mathscr{F}$. We then denote by $\mathrm{H}_{f}(\theta) \triangleq \mathrm{H}(2 \pi f j, \theta)$.

The identification procedure should be designed to process two types of data:

- estimated transfer $H_{f}$

- signals in the frequency domain for the test excitation $u_{f}$ and the measurements $Y_{f}$.

We can show that these two alternatives can be expressed in a single formalism as follows :

Find the values $\theta$ which minimize:

$$
J(\theta)=\sum_{f \in \mathscr{F}}\left\|\mathbf{H}_{f}-\mathrm{H}_{f}(\theta)\right\|_{W_{f}}^{2}
$$

Depending on the type of data processed, the variable $\mathbf{H}_{f}$ and the diagonal weighting matrix $W_{f}=\operatorname{diag}\left(w_{l, f}\right)$ take the following values :

- Identification on estimated transfer:

$$
\mathbf{H}_{f}=H_{f} \quad w_{l, f}=c_{l} \chi_{l, f}
$$

where $c_{l}$ is a scaling factor and $\chi_{l, f}$ is a quality indicator for the $l$ th component of the estimated transfer.

- Identification on measurements: 


$$
\mathbf{H}_{f}=\frac{Y_{f}}{u_{f}} \quad w_{l, f}=c_{l}\left|u_{f}\right|
$$

where again $c_{l}$ is a scaling factor

Into order to avoid any confusion, in the remainder of this article we use the term "transfer" instead of "measurement" to designate the data being processed.

\subsection{Minimisation techniques}

The criterion 3 is non linear in the parameters $\theta$. Two main methods are available for this minimization problem.

2.2.1. Iterative least squares (ILS) (Sanathanan and Koerner, 1963)

This method consists in adopting for the denominator of $\mathrm{H}_{f}(\theta)$ responsible for the non-linearity the values $\tilde{\mathrm{d}}_{f}$ computed at the preceding iteration. The problem can then be recast in a conventional least squares form

$$
J_{i}(\theta)=\sum_{f \in \mathscr{F}}\left\|\mathrm{d}_{f}(\theta) \mathbf{H}_{f}-\mathrm{N}_{f}(\theta)\right\|_{\frac{W_{f}}{\left|\mathrm{~d}_{f}\right|}}^{2}
$$

The great advantage for this method is that it offers an elegant way to initialize the minimisation simply by taking $\tilde{\mathrm{d}}_{f}=1$ at the first iteration.

\subsubsection{Gauss-Newton algorithm (GN) 1987)}

(Whitfield,

The Gauss-Newton approach consists in linearizing the function $\mathrm{H}_{f}(\theta)$ about the previous value $\tilde{\theta}$ of the parameters. In the case considered here, the equations simplify to

$$
J_{i}(\theta)=\sum_{f \in \mathscr{F}}\left\|\Delta \mathbf{H}-\left(\frac{\mathrm{N}_{f}(\theta)}{\tilde{\mathrm{d}}_{f}}-\frac{\tilde{\mathrm{N}}_{f}}{\tilde{\mathrm{d}}_{f}^{2}} \mathrm{~d}_{f}(\theta)\right)\right\|_{W_{f}}^{2}
$$

where $\Delta \mathbf{H}=\mathbf{H}_{f}-\tilde{\mathrm{H}}_{f}$.

These method provides a more accurate convergence but it requires relatively precise initial values for $\tilde{\mathrm{N}}_{f}$ and $\tilde{\mathrm{d}}_{f}$. A nice way to deal with this difficulty is to use both algorithms in combination starting by a few iterations of ILS followed by an optimization refinement performed with the GN technique.

\section{COMPUTING THE LEAST SQUARES SOLUTION}

In this section, we describe how to compute efficiently the real solution of the complex least squares problems of equations 6 and 7 . We first use a conventional polynomial basis. Then, as the numerical sensitivity reveals to be a critical issue, we adopt more appropriate bases.

\subsection{Conventional polynomials}

Both ILS and GN techniques can be recast in solving, in the least squares sense, an overdetermined system of the form:

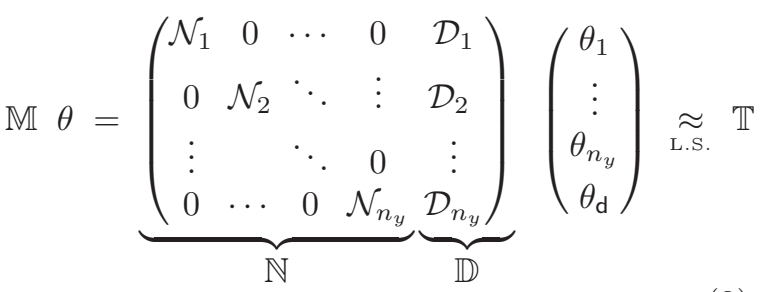

where the blocks $\mathcal{N}_{l}$ and $\mathcal{D}_{l}$ further decompose into

$$
\mathcal{N}_{l}=\left[\begin{array}{cc}
\mathcal{N}_{l}^{\mathrm{e}} & 0 \\
0 & \mathcal{N}_{l}^{\mathrm{o}}
\end{array}\right] \quad \text { and } \quad \mathcal{D}_{l}=\left[\begin{array}{ll}
\mathcal{D}_{l}^{\mathrm{ee}} & \mathcal{D}_{l}^{\mathrm{eo}} \\
\mathcal{D}_{l}^{\mathrm{oe}} & \mathcal{D}_{l}^{\mathrm{oo}}
\end{array}\right]
$$

corresponding to a subdivision of the coefficients $\theta_{l}$ and $\theta_{\mathrm{d}}$ into the coefficients relative to the even and the odd powers of the polynomials. The subblocks of the numerator are equal to

$$
\begin{aligned}
& \mathcal{N}_{l}^{\mathrm{e}}=\mathcal{W}_{\mathrm{n}, l} \Omega^{\mathrm{e}} \\
& \mathcal{N}_{l}^{\mathrm{o}}=\mathcal{W}_{\mathrm{n}, l} \Omega^{\mathrm{o}}
\end{aligned} \quad \mathcal{W}_{\mathrm{n}, l}=\underset{f}{\operatorname{diag}}\left(w_{l, f} /\left|\tilde{\mathrm{d}}_{f}\right|\right)
$$

while, for the denominator, we have

$$
\begin{aligned}
& \mathcal{D}_{l}^{\mathrm{ee}}=-\operatorname{Re}\left(\mathcal{W}_{\mathrm{d}, l}\right) \Omega^{\mathrm{e}} \quad \mathcal{D}_{l}^{\mathrm{oe}}=-\operatorname{Im}\left(\mathcal{W}_{\mathrm{d}, l}\right) \Omega^{\mathrm{e}} \\
& \mathcal{D}_{l}^{\text {oo }}=-\operatorname{Re}\left(\mathcal{W}_{\mathrm{d}, l}\right) \Omega^{\mathrm{o}} \quad \mathcal{D}_{l}^{\mathrm{eo}}=\operatorname{Im}\left(\mathcal{W}_{\mathrm{d}, l}\right) \Omega^{\mathrm{o}} \\
& \mathcal{W}_{\mathrm{d}, l}= \begin{cases}\underset{f}{\operatorname{diag}}\left(w_{l, f} \mathbf{H}_{l, f} /\left|\tilde{\mathrm{d}}_{f}\right|\right) & \text { ILS } \\
\underset{f}{\operatorname{diag}}\left(w_{l, f} \tilde{\mathrm{N}}_{l, f} /\left|\tilde{\mathrm{d}}_{f}^{2}\right|\right) & \mathrm{GN}\end{cases}
\end{aligned}
$$

The expression of the matrices $\Omega^{\mathrm{e}}$ and $\Omega^{\circ}$ depends on the powers of the pulsations $\omega$ corresponding to the frequencies in the set $\mathscr{F}(\omega=2 \pi f)$.

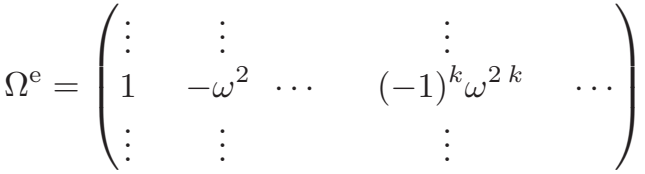

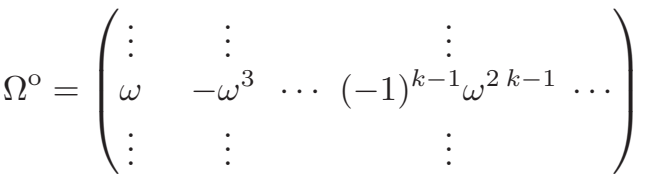

These matrices $\Omega^{\mathrm{e}}$ and $\Omega^{\circ}$ are the causes of numerical problems. A simple glance at equations 12 and 13 , shows that, as the power value $k$ increases, the dispersion of the matrix components increases tremendously leading to unfavourable conditioning. In order to identify high order systems, it is necessary to consider other polynomial bases that are numerically less sensitive. 


\subsection{Optimal polynomial bases}

Each polynomial of the model transfer function $\mathrm{H}(s, \theta)$ can be expressed in a specific polynomial basis according to

$$
\begin{aligned}
\mathrm{d}(s) & =\sum_{k=0}^{n_{d}} \mathrm{p}_{\mathrm{d}, k}(s) \quad \theta_{\mathrm{d}, k} \\
\mathrm{~N}_{l}(s) & =\sum_{k=0}^{n_{d}} \mathrm{p}_{l, k}(s) \quad \theta_{l, k}
\end{aligned}
$$

where $\left(\mathrm{p}_{l, k}(s), k=1, \ldots, n_{d}\right)$ are polynomial series of increasing degree $k$.

We shall consider only the bases that respect parity, i.e. the polynomials $\mathrm{p}_{l, k}(s)$ are even when the degree $k$ is even and odd when $k$ is odd. In that case, the formulation of the least squares problem is quite similar the one described above except the matrices $\Omega^{\mathrm{e}}$ and $\Omega^{\circ}$ (equations 12 and 13). For these matrices, it suffices to replace the powers $\omega^{k}$ by $\mathrm{p}_{l, k}(\omega)$.

Inspired by the pioneer work of G. Forsythe (Forsythe, 1957), it is possible to build polynomial bases (Rolain et al., 1995) respecting parity and particularly appropriate for this problem since they make orthonormal the blocks $\mathcal{N}_{l}$ and the last $n_{d}$ columns $\mathbb{D}$ in equation 8 . This can be accomplished by defining $\mathrm{p}_{l, k}(s)$ according to the following recursion

$$
\begin{aligned}
\tilde{\mathrm{p}}_{l, k}(s) & =s \mathrm{p}_{l, k-1}(s)+Z_{k-1} \mathrm{p}_{l, k-2}(s) \\
Z_{k} & =\sqrt{\sum_{f}\left|w_{l, f} \tilde{\mathrm{p}}_{l, k}(2 \pi f j)\right|^{2}} \\
\mathrm{p}_{l, k}(s) & =\frac{\tilde{\mathrm{p}}_{l, k}(s)}{Z_{k}}
\end{aligned}
$$

where $w_{l, f}$ are the components of $\mathcal{W}_{\mathrm{n}, l}$ (equation 10). A similar recursion can be written for the basis of the denominator.

The favourable impact on numerical robustness induced by these polynomial bases is quantified by the condition number of the least square matrix $\mathbb{M}$ (equation 8). Since the main blocks $\mathbb{N}$ and $\mathbb{D}$ are then orthonormal, it can be shown that the condition number of $\mathbb{M}$ only depends on the minimum angle $\alpha_{\min }$ between the subspaces spanned by the columns of $\mathbb{N}$ and $\mathbb{D}$. It is equal to

$$
\kappa(\mathbb{M})=\cot \left(\alpha_{\min } / 2\right)
$$

The subspaces spanned of $\mathbb{N}$ and $\mathbb{D}$ are independent of the polynomial bases. They are inherent to the definition of the least squares problem at each iteration. Hence the polynomial bases defined by the recursion 16 allow to reach the value 17 which is geometrically defined and by such constitutes a minimum value.

At this stage, it must be emphasized that the use of these polynomial bases was only made possible by the specific formulation of the minimization algorithms into the form of a least squares problem (equation 6 and 7). Moreover, a careful implementation taking full advantage of the block structure of the matrix $\mathbb{M}$ and of the orthonormality of its blocks produces an algorithm particularly efficient and extremely resistant from a numerical point of view.

\section{MONITORING THE IDENTIFICATION}

\subsection{Automatic order determination}

4.1.1. The approach adopted Based on the experience accumulated by processing numerous flight tests, we develop an heuristic approach for computing the best value for the system order $n_{d}$.

We begin by carrying out a first identification ab nihile with an overestimated guess for $n_{d}$. At this stage, we use the ILS technique to compute the first identified model.

Based on this model, a systematic analysis is then started in order to discard undesirable or meaningless modes. The modes are scanned to check whether one or more comply with the following criteria:

- unstable

- real mode or over-damped mode

- weak contribution on the optimisation criterion

- in the close neighbourhood of another mode

If so, an optimisation phase is launched initialized with a model where the suspected mode has been removed.

To confirm the suppression of this mode, two types of criteria are evaluated on the new model : a global relative criterion defined by 18 and local criteria for each transfer (relation 19).

$$
\begin{gathered}
\Gamma=\sqrt{\frac{\sum_{f}\left\|\Delta \widehat{\mathbf{H}}_{f}\right\|_{W_{f}}^{2}}{\sum_{f}\left\|\mathbf{H}_{f}\right\|_{W_{f}}^{2}}} \\
\gamma_{l}=\sqrt{\frac{\sum_{f}\left|w_{l, f} \Delta \widehat{\mathbf{H}}_{l, f}\right|^{2}}{\sum_{f}\left|w_{l, f} \mathbf{H}_{l, f}\right|^{2}}}
\end{gathered}
$$

where $\Delta \widehat{\mathbf{H}}_{f}=\mathbf{H}_{f}-\mathrm{H}_{f}(\widehat{\theta})$ and $\widehat{\theta}$ is the optimum value of the parameters. The criteria $\gamma_{l}$ proved to be useful to prevent undue elimination of local modes which are only visible on a few transfers.

If the optimization with the reduced model does not result in a significant increase (specified by a threshold) of any of the above criteria, the suppression of the mode is confirmed and this elimination process is continued with the new model. Otherwise the suspected mode is retained. 
This process is continued until no more candidate for elimination is found.

4.1.2. Checking the process We developed a graphic tool dubbed PtiRonds (figure 1) to check the validity of the above process for order determination. On the left part, the position of the identified modes are plotted in a damping ratio versus frequency system of axes. The upper graphic is full-scaled while the lower is a zoomed view on the zone of interest. They provide information about the stability of the modes as the order of the identified system $n_{d}$ is reduced in the procedure.

The table on the right hand side contains the characteristics of the modes as well as an indication of the modes cancelled at each stage of the procedure. The corresponding evolution of the global criterion (equation 18) is depicted above this table.

\subsection{Analysis of the identified model}

We developed a graphical interface named Pave$\mathrm{Bleu}$ for the analysis of several properties of the identified model.

4.2.1. Modal contributions The central and main part of the display is related to the contribution of the modes on each transfer. The identified modes appear on the horizontal axis. They are ordered according to their frequency on a logarithmic scale. The transfers are referenced by a ten digit number. Their contribution are depicted by the size of the cyan rectangles. Their width is related to the frequency spread of each mode about its resonance which is equal to $2 \zeta f_{n}\left(f_{n}\right.$ is the natural frequency, $\zeta$ its damping ratio). For each transfer, the heights of the rectangles are equal to the square root of the proportion of energy due to each mode relative to the energy of the whole system on this transfer.

As the modes get closer, they might be some severe overlapping between the rectangles. In order to clarify the user's analysis, the interface incorporate a few interactive mechanisms : rescaling of the frequency axis, buttons for masking contributions, width reduction for the contribution of overdamped modes (in red on the graphic), minimal threshold for contribution visibility.

4.2.2. Modal relevance The lower part of the graphic represents with green rectangles the relevance of each mode. It is defined by the increase on the global criterion 18 that would result from the suppression of the mode in the identified model. For comparison purpose, the relative size of the global criterion appears on the left of this diagram (the red rectangle).
4.2.3. Quality of transfer fitting The blue rectangles on the left depicted how much of the energy on each transfer originates from the identified model. Their height is then given by $1-\gamma_{l}$ (equation 19).

4.2.4. Averaged transfer weighting The yellow rectangles on the left correspond to the means over the frequencies $f$ of the quality factor in the weighting $w_{l, f k}$ (equation 4 and 5 ). They reveal how much each transfer is accounted for in the identification.

\section{CONCLUSION}

In this article, we first presented the identification technique that we developed. We showed that, at each iteration, it can be recast in the form of a least squares problem. This formulation is essential because we can then compute optimal polynomial bases for defining the model. These bases evolve at each iteration of the optimisation in a self-adapting process hence ensuring the best numerical conditions throughout the procedure of identification. Hence, at each loop of this method, two updates are performed simultaneously: one on the polynomial bases, the other on the identified parameters.

The last part of this article is relative to the embedding of the identification routines that were necessary to integrate them into the operational environment of flight tests. Adequate and friendly interfaces proved to be essential for the acceptance of these new tools and for an efficient exploitation of the results.

\section{REFERENCES}

Forsythe, George E. (1957). Generation and use of orthogonal polynomials for data-fitting with a digital computer. J. Soc. Indust. Appl. Math. 5(2), 74-88.

Rolain, Y., R. Pintelon, K. Q. Xu and H. Vold (1995). Best conditioned parametric identification of transfer function models in the frequency domain. IEEE Transactions on Automatic Control 40(11), 1954-1960.

Sanathanan, C. K. and J. Koerner (1963). Transfer function synthesis as a ratio of two complex polynomials. IEEE Transactions on Automatic Control 9(1), 56-58.

Welch, P. D. (1967). The use of fast Fourier transform for the estimation of power spectra: A method based on time averaging over short, modified periodograms. IEEE Trans. Audio Electroacoust. AU-15, 70-73.

Whitfield, A.H. (1987). Asymptotic behaviour of transfer function synthesis methods. International Journal of Control 45(3), 1083-1092. 

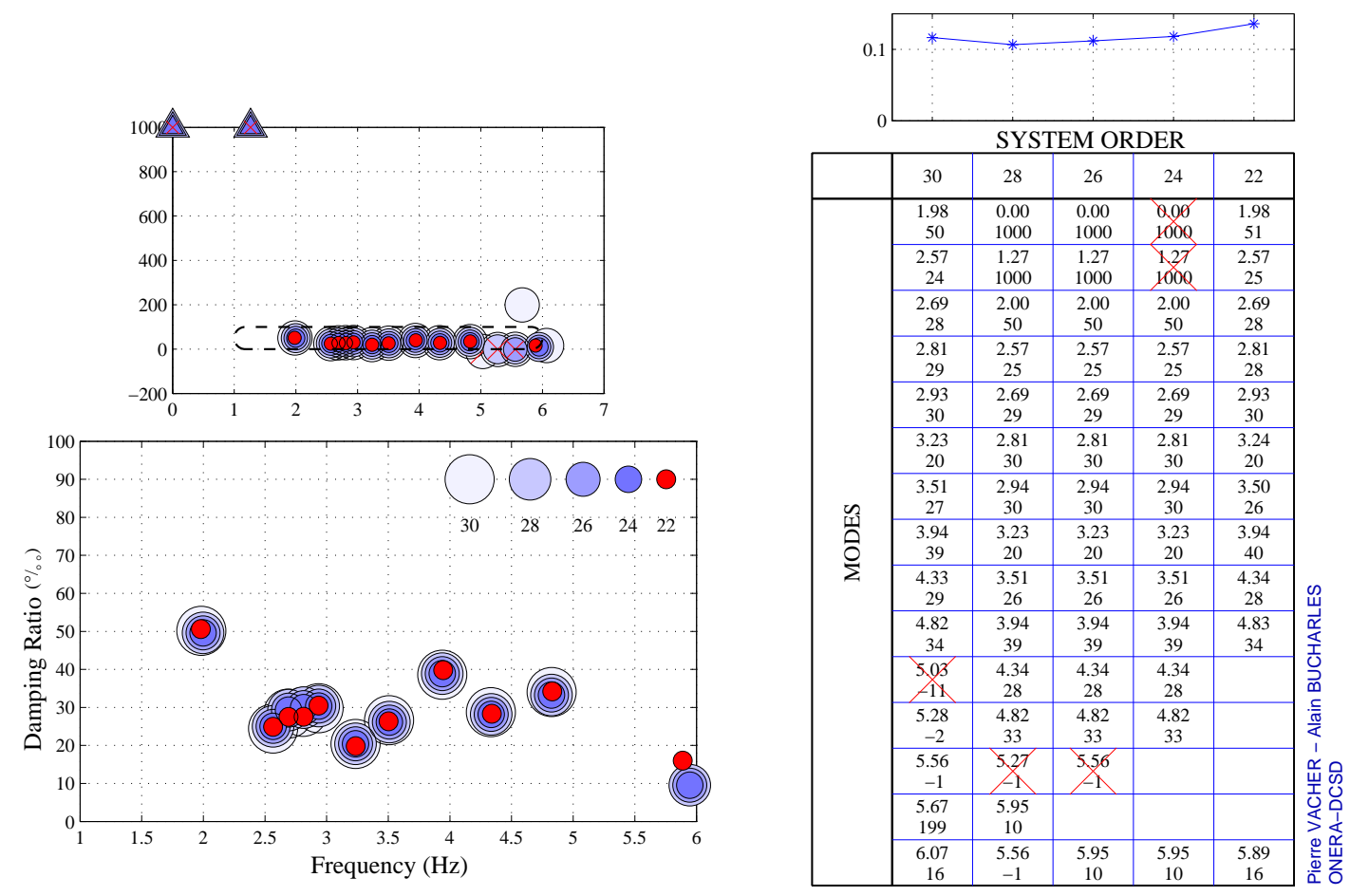

Fig. 1. Interface PtiRonds: validation of order determination

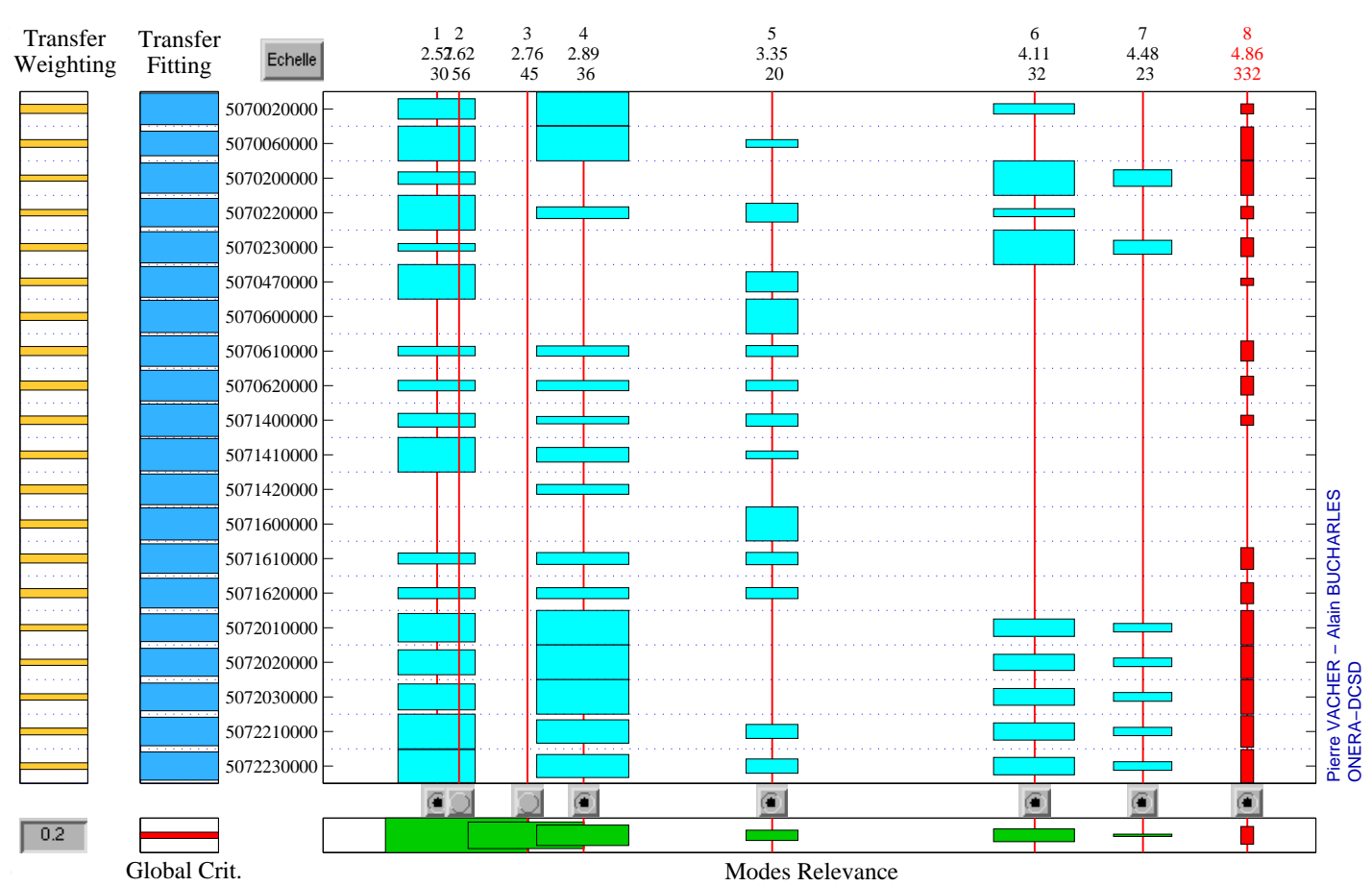

Fig. 2. Interface PaveBleu : analysis of the identified model 